\title{
Assessing Advance Care Planning in Individuals with Lynch Syndrome
}

\author{
Victoria Fenton $^{1} \cdot$ Lauren Fletcher $^{1} \cdot$ Jennifer Bowles $^{1} \cdot$ Kelsey Hennig $^{2} \cdot$ Allison M. Burton-Chase $^{1}$ (D)
}

Accepted: 1 January 2021 / Published online: 14 January 2021

(C) The Author(s), under exclusive licence to Springer Nature Switzerland AG part of Springer Nature 2021

\begin{abstract}
Lynch syndrome (LS) is a hereditary cancer syndrome characterized by an increased risk of multiple cancers, predominantly endometrial and colorectal, at a younger age (typically < 50). In prior research, high death anxiety and a lack of provider-initiated communication about advance care planning (ACP) have been shown to decrease a patient's likelihood of having advance directives. Providers often have gaps in knowledge and are uncomfortable with these conversations. We used a mixed methods approach (quantitative survey with a follow-up telephone interview) to assess knowledge, preferences, and attitudes regarding ACP in individuals with LS $(n=20)$. This study also assessed which ACP documents individuals already had in place and which persons (providers, family, or friends) an individual made aware of the documentation and/or preferences. These data were analyzed to determine patient preferences for who is responsible for initiating these conversations, identify motivating factors and barriers to these conversations, and determine whether the current conversations are adequate to meet the needs of this patient population. Participants recognized the importance of ACP and expressed interest in creating these documents. However, knowledge and confidence about these topics were lacking, with many participants attributing this to their young age and lack of experience. Although uncomfortable, many patients want to have ACP discussions with their providers, but frequently patients were only asked if these documents are completed with no further discussion. These findings can inform educational efforts to improve knowledge of ACP and interventional research to increase use of ACP by individuals with LS.
\end{abstract}

Keywords Lynch syndrome $\cdot$ Advance care planning $\cdot$ Patient communication $\cdot$ Family communication

\section{Introduction}

Lynch syndrome (LS) is a hereditary cancer syndrome caused by germline mutations in the DNA mismatch repair genes (PMS2, MSH6, MLH1, and MSH2) or a deletion of the EPCAM gene. LS is characterized by a predisposition to certain cancers, predominately colorectal, endometrial, ovarian, and gastric. Other associated cancers include, but are not limited to, stomach, urothelial tract, small bowel, pancreas, biliary tract, and sebaceous skin [1]. The risks of developing

This article is part of the Topical Collection on Medicine

Allison M. Burton-Chase

allison.burton-chase@acphs.edu

1 Department of Population Health Sciences, Albany College of Pharmacy and Health Sciences, 106 New Scotland Avenue, Albany, NY 12208, USA

2 School of Pharmacy and Pharmaceutical Sciences, Binghamton University, Binghamton, NY, USA colorectal, endometrial, and ovarian cancer are described in Table 1 [2]. In addition to the significantly increased lifetime risk of developing these cancers, individuals with LS are more likely to develop cancer at a younger age. The average age of onset of these cancers in individuals with LS is 45 [3]. Recent estimates indicate that about 1 in 279 people in the general population could have a LS mutation [1].

Advance directives, mainly consisting of health care proxies and living wills, are documents that can be used to help guide end-of-life decision-making if individuals are unable to make decisions for themselves. In a longitudinal cohort study that analyzed associations between end-of-life discussions, patient mental health, and bereavement adjustment, Wright et al. reported that individuals who expressed their wishes using these documents had lower rates of ventilation, resuscitation, and intensive care unit (ICU) admission, while also having earlier hospice enrollment and increased quality of life. Completing these documents was not associated with increased feelings of worry in patients; however, it was associated with improved bereavement adjustment in caregivers [4]. 
Table 1 Cancer risks by mutation [2]

\begin{tabular}{lllll} 
Cancer type & General population & MLH1\&MSH2 & MSH6 & PMS2 \\
\hline Colorectal & $5.5 \%$ & $30-74 \%$ & $10-22 \%$ & $15-20 \%$ \\
Endometrial & $2.7 \%$ & $54 \%$ & $71 \%$ & $15 \%$ \\
Ovarian & $1.6 \%$ & $4-20 \% *$ & $4-20 \% *$ & $4-20 \% *$
\end{tabular}

*The most recent studies have not yet determined mutation differences in ovarian cancer

Despite the benefits, these documents are underutilized in the general population [5]. A systematic review of studies published from 2011 to 2016 found that only $36.7 \%$ of adults in the USA have completed an advance directive [5]. This percentage remained relatively constant among all years reviewed, and there was no significant difference between healthy adults and those with chronic illnesses. The only significant factor that predicted whether an individual would create an advance directive was their age. Adults over the age of 65 had a significantly higher rate of completing advance care directives [5].

Advance care planning (ACP) documents are underused in the general population, but individuals with LS may face additional barriers when completing these documents. The finding in the literature that older age, rather than diagnosis, is associated with higher rates of completing advance care directives may indicate that the younger age at diagnosis for individuals with LS creates a barrier for ACP. In addition to age as a barrier, the literature also shows that there are communication barriers between individuals with LS patients and their providers [6]. Information often gets lost between different health care providers, and these providers are sometimes unsure of what their specific roles entail [6]. There is not currently a clear, universal decision regarding who is responsible for initiating the conversation about ACP with individuals with LS.

The goal of this study was to assess knowledge and utilization of ACP in individuals with LS through evaluating their current level of knowledge and confidence in that knowledge, as well as determining which documents an individual had in place. Additionally, this study aimed to evaluate attitudes and preferences with regards to these documents, and to identify barriers that may be preventing individuals with LS from having ACP conversations with their providers and completing the associated documentation. To the best of our knowledge, this is the first study that examines ACP in individuals with LS. Furthermore, we were unable to identify any literature that examined ACP in individuals with other hereditary cancer syndromes, including, but not limited to, hereditary breast and ovarian cancer syndrome and Li-Fraumeni syndrome.

\section{Methods}

This mixed methods study was approved by the Albany College of Pharmacy and Health Sciences (ACPHS) Institutional Review Board. Participants $(n=20)$ were recruited through the Lynch Syndrome International (LSI) Facebook page. The Facebook page was utilized to create and share a post that provided the eligibility criteria and study details. Patients were eligible to participate in the study if they met the following criteria: (1) 18 years or older, (2) able to speak and read English, (3) able to be contacted by phone or email, (4) have undergone genetic counseling and testing for LS, and (5) tested positive for a LS mutation. The research team recruited the first 20 participants who responded to the Facebook post either by phone or email for surveys and indepth telephone interviews. All of those first 20 participants met the eligibility criteria, were consented, and completed the survey; 18 participants subsequently completed the follow-up interview. Participants received a $\$ 10$ Amazon gift card as a thank you for completing the survey and a second one upon completion of the interview. Recruitment methods were similar to what were used in a prior study, described in detail in another paper [7].

\section{Data Collection}

All participants were given access to the online survey link via REDCap (http://www.project-redcap.org/), a browser-based, electronic data capture software package compliant with HIPAA restrictions [8]. Participants were asked about their LS mutation, cancer history, ACP, and demographic information. Cancer history included both personal and family history. Questions about ACP included rating how knowledgeable and confident participants felt about ACP, as well as their current completion of and preferences regarding ACP. The survey assessed which ACP documents participants had completed, who this information had been disclosed to, participant interest in learning more, and levels of death anxiety. A glossary was provided that defined the ACP terms used within the survey questions. The demographic information gathered from the survey included sex, age, current marital status, children, ethnic/racial background, education, employment, average annual income, and current household financial situation.

A scheduled phone call was conducted within 2 weeks of completing the REDCap survey. The telephone interview was designed to elicit a better understanding of the participant's opinions based on their experiences with health care providers, and to give the participants the opportunity to elaborate on their answers in the survey. During the interview, participants were asked about their knowledge of, experience with, and attitudes toward $\mathrm{ACP}$, along with their preferences in how to be informed about ACP. For example, participants were asked questions including 
"What exposure have you had to ACP so far?" and "Can you tell me a little more about why you prefer an oncologist to initiate conversations about ACP?" Each interview was recorded and then transcribed for data analysis.

\section{Data Analysis}

Once data collection was concluded, the transcribed interviews were analyzed using a grounded theory approach [9]. Two authors ( $\mathrm{KH}$ and $\mathrm{ABC}$ ) reviewed the interview guide and randomly selected three interviews to create a preliminary codebook. Three authors (JB, LF, and VF) applied the codebook to randomly selected transcripts to establish inter-coder reliability. Once an inter-coder reliability of greater than $80 \%$ was achieved, the transcripts were randomly assigned to the three authors (JB, LF, and VF) to be analyzed using the codebook. The codebook was modified and refined through analyzing the transcripts. The coded transcripts were then randomly assigned to the three authors for a second review. Members of the research team (JB, LF, VF, and ABC) met multiple times to review the coded transcripts and make final decisions about coding.

\section{Results}

Participant demographics $(n=20)$ are listed in Table 2. Eleven participants $(61 \%)$ had a health care proxy, and 7 participants (39\%) did not have a health care proxy. Seven participants (39\%) had a living will in place, $10(56 \%)$ did not have a living will in place, and $1(6 \%)$ participant had a living will in progress. Eleven participants (61\%) expressed interest in learning more about ACP. If given the opportunity, 11 participants (61\%) also said they would create ACP documents.

Figure 1 shows how participants received information about ACP. In contrast, Fig. 2 shows how participants would prefer to receive this information. There was a disparity in how participants received information versus how they wished to receive information. Most of the participants were informed about ACP by personal and family experiences. They reported learning about ACP from experiences such as witnessing the impact that not having these conversations and documents can have on end-of-life experiences. Three participants $(17 \%)$ shared that they wished to have guidance in developing and completing ACP documents. Participants reported interest in one-on-one instruction as well as sample copies of completed ACP documents. One participant summarized the desires of many when he/she remarked "...they always tell me at the hospital...you can pick up some information on your way out and I mean frankly a hospital is probably the last place I [want to] get information [because] I just [want to] get in and get out as fast as possible.”
Table 2 Participant demographics

\begin{tabular}{ll}
\hline Characteristic & $\%(n)$ \\
\hline Mean age (range), years & $48.2(29-70)$ \\
Gender, female & $85(17)$ \\
Race, White & $95(19)$ \\
Education, greater than high school education & $95(19)$ \\
Income, greater than $\$ 25,000$ per year & $94.7(18)$ \\
Married & $85(17)$ \\
Cancer history:* & \\
Previvor & $45(9)$ \\
Survivor & $55(11)$ \\
Colorectal cancer & $30(6)$ \\
Endometrial cancer & $15(3)$ \\
Ovarian cancer & $15(3)$ \\
All other cancers & $20(4)$ \\
Geographic location: & \\
Eastern time zone & $45(9)$ \\
Central time zone & $40(8)$ \\
Mountain time zone & $10(2)$ \\
Pacific time zone & $5(1)$ \\
\hline
\end{tabular}

*Participants may have had multiple cancers, so the percentages may not sum to 100

Three participants (17\%) reported having a conversation with a health care provider (HCP) about their ACP documents, 8 participants $(44 \%)$ did not report having a conversation with a HCP, and 1 participant (6\%) was unsure. Six participants (33\%) recalled having a HCP ask about ACP documents, but no further conversation occurred. Younger participants, in particular, reported not being asked about advance directives. The majority of participants (61\%) wished to discuss ACP with a HCP. Of this $61 \%$, only 4 participants ( $22 \%$ ) noted that they wanted a HCP to initiate this conversation. When asked if the participant would initiate the conversation if his or her HCP did not, one participant replied “. . . as far as the primary care doctor, I don't know if I would initiate it with her just because...normally our meetings are...a physical and that's about it." Six participants (33\%) expressed that they wanted to have a pre-existing, trusted relationship with the HCP with whom they discussed ACP documents. One participant wished to speak to a counselor/social worker, and did not feel it was as appropriate to speak with a HCP about ACP. Participants reported that a key factor in conversations about ACP is comfort with the HCP. The provider specialty (e.g., surgeon, oncologist, $\mathrm{PCP}$ ) was not an important factor in whether participants wanted to have this conversation with a HCP, rather it was their level of trust in the provider and the nature of the patient-provider relationship. One participant summarized this by stating, "...It probably should be your primary care physician but...it depends on who you personally feel you have the best relationship with." 


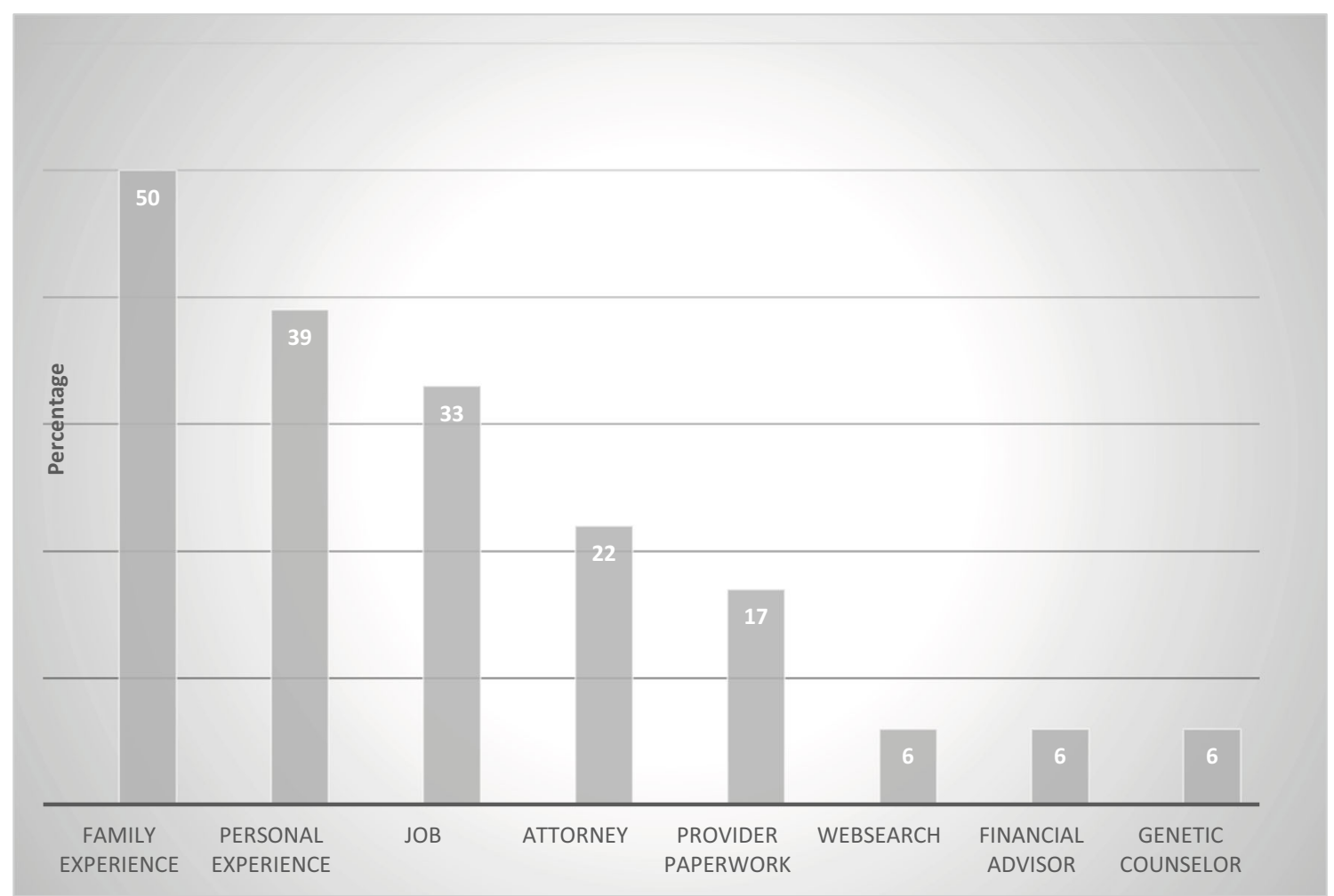

Fig. 1 How individuals with LS receive information about ACP

Although participants frequently felt uncomfortable discussing end-of-life preferences, they recognized the importance of having these conversations. Regarding discussing her preferences with her husband, who also was her health care proxy, one participant shared: "My husband wasn't thrilled about it. He kind of got up from the table. I think my parents and I are much more comfortable....we are just fortunate that we can have these conversations. I don't expect everybody to be that way. It's certainly not comfortable but...it has to be discussed."

Major motivations, perceived benefits, and perceived barriers are outlined in Table 3. Thirteen participants (72\%) perceived a major benefit of ACP is knowing someone's wishes. Four participants $(22 \%)$ viewed the preservation of quality of life as a major benefit of ACP.

For many participants, LS was not the major motivation for creating ACP documents. One participant put it best when he/she said "Lynch syndrome really hasn't affected me as far as the advance directives, we were working on those before we even knew about Lynch syndrome." When one participant was asked what has kept him/her from creating advance care directives, he/ she simply replied "life."

Participants knew they needed advance directives. When presented with the statement "discussing my preferences for lifesustaining treatments with my family would only lead to disagreement and conflict," 16 participants $(88.9 \%)$ disagreed with the statement, 1 participant (6\%) agreed, and 1 participant was unsure $(6 \%)$. Participants also believed even those in good health needed to think about ACP, with 17 participants (94\%) disagreeing with the statement "people in good health do not need to think about preparing a living will." Most participants acknowledged the uncertainty of life. One participant summarized the thoughts of many when he/she said, "I think that everybody needs a living will. I don't care if you're in good health or not, you never know what tomorrow will bring...[it] is not guaranteed for anybody."

Participants understood that these documents should be completed and reconsidered throughout their lives, not just after diagnosis with a life-threatening medical condition. Most participants had limited knowledge about advance directives and end-of-life planning. When asked about their knowledge of advance care directives, 4 participants $(22 \%)$ described themselves as extremely knowledgeable, 10 participants $(56 \%)$ as somewhat knowledgeable, and 4 participants (22\%) as not at all knowledgeable. Similarly, when asked how confident they felt in their ACP knowledge, 4 participants (22\%) felt extremely confident, 10 participants (56\%) felt somewhat confident, and 4 participants (22\%) felt not at all confident in their knowledge of advance care directives. A majority of the participants who were knowledgeable and confident in their knowledge had a job in the health care field or a close family member with a job in the health care field.

\section{Discussion}

Individuals with LS recognize the importance of ACP and have an interest in completing these documents. Our results confirm 


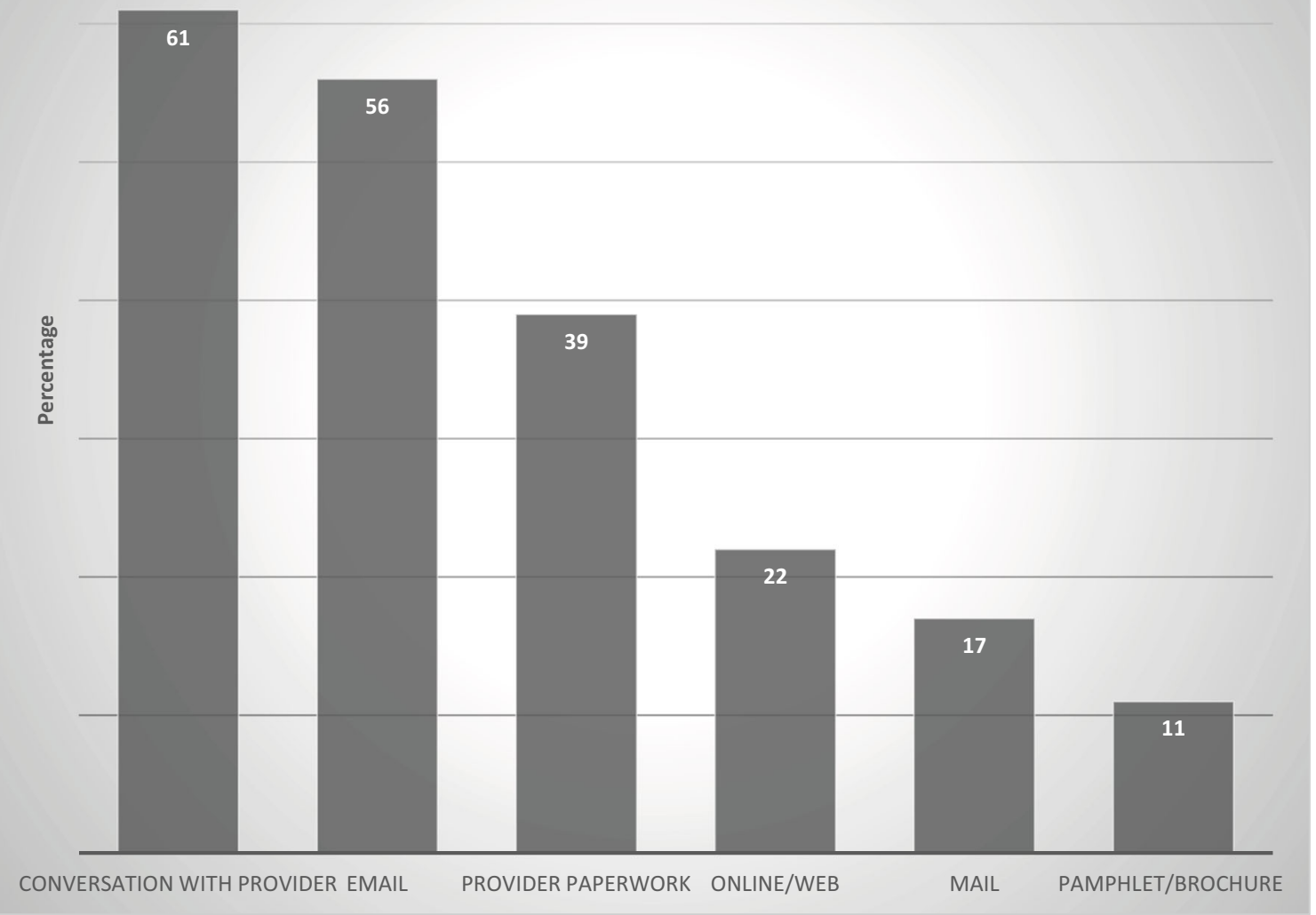

Fig. 2 How individuals with LS would like to receive information about ACP

that barriers to ACP include a lack knowledge and confidence in ACP topics, mainly due to young age and minimal experience with ACP. Even though participants admitted to feeling uncomfortable discussing end-of-life preferences, they recognize the importance of having these conversations with both providers and family members. A diagnosis of LS was only one of multiple factors associated with participant motivation to complete ACP documents and agreed that this documentation was important regardless of their diagnosis of LS.

All people benefit from creating and discussing ACP documents. The necessity of these documents is illuminated by what occurs when they are not in place. When these conversations with a health care proxy or provider do not take place, no one is made aware of an individual's wishes. In turn, the end-of-life care that a patient receives may not be in line with their wishes. Further, the default end-of-life care for individuals who lack ACP documents is life-sustaining measures, which equate to more demand on health care workers and more health care dollars spent. The cost of keeping a patient on life support in the ICU is minimally estimated to be between $\$ 2000$ and $\$ 4000$ per day, but can cost upwards of hundreds of thousands per year depending on a patient's condition [10]. The ratio of benefit to harm with treatment is often skewed. For example, with cardiopulmonary resuscitation (CPR) a patient may be left worse off than before, with cracked ribs or damage to internal organs [11]. Additionally, the treatment may ultimately be futile, with no change in the outcome, including death. With ACP, these measures are often limited, ultimately saving time, money, and energy [11].

Participants who had a family experience with ACP reported being aware of their loved ones wishes, which has been linked to decreased feelings of grief and stress after the death of a loved one [12]. One randomized, controlled trial found that patients with ACP documentation were more likely to have their wishes known, and their family members experienced significantly lower stress, anxiety, and depression after their loved one had died [12]. Similarly, a longitudinal study of patients with advanced cancer and their informal caregivers found that end-of-life discussions did not increase rates of major depressive disorder or feelings of worry [4]. Additionally, these discussions were associated with lower rates of ventilation, resuscitation, ICU admission, and earlier hospice enrollment [4]. One of the benefits of ACP lies with being proactive rather than reactive, thereby reducing the undesirable feelings and consequences that are often associated with end-of-life decision-making. 
Table 3 Perceived barriers to, benefits of, and motivations for completing ACP documents for individuals with LS

Perceived barriers
Discomfort with the
conversation
Lack of time

Perceived benefits

Family being aware of preferences

Preserving quality of life

Participant motivations

Having children

New diagnosis

Older age

Prior family experience

Spouse (especially wife)
Quote(s)

I just think some people might have a differing opinion about things and...I don't like conflict.

It's just that it hasn't been a priority. I guess over the last two years that the priority was just...surviving and getting through surgery and...then chemo...then getting through genetic testing and getting everybody else in my family tested and so it's those things that [have]...taken priority.

When you have children, you kind of just end up being in survival mode some days and these larger existential life issues don't come to the foreground until they have to. Which is a bad way of looking at it but kind of how we've been doing it. So no, there is nothing stopping me other than I should just do it.

Quote(s)

I think it was helpful to have it in writing because...in those moments if he were... unable to speak for himself, I'm sure everybody is [going to] be freaking out. And so I think it's good for those moments to have something concrete and tangible.

My sister passed suddenly and nobody really knew what she wanted. Nobody really knew what the right thing was to do...everybody second guessed what they should have done. [They] painstakingly tried to make decisions... and could not come together, and it was hard for them. And then my mom died four months later and it was a lot easier [because she had these documents in place]. So I have seen it on both sides.

I feel strongly... [that] people [should not be] suffering on a ventilator. I feel very strongly about quality of life.

Quote(s)

To make sure our son was protected and taken care of. Well we did it originally when my son was young...to make sure that there was no questions about... who would care for him in the event that something would happen to us.

Were I [to be] diagnosed with a stage of cancer where my...prognosis was poor, then I think that's the time to have that discussion with the provider. The family knows what's going on....[and] you want everybody on board.

Even with my whole family in the health care industry... and being in the health care industry myself, it just... is one of those things that as you get older, you need it.

I think if none of us had ever had cancer, we probably wouldn't be having these conversations.

Well I think initially it is just good communication...my husband and I both lost our fathers to cancer and his my mother-in-law also passed away. We both lost brothers to cancer and so it's not... a stranger to our family. So we address these things very directly.

My wife was more involved...than I was. I just follow her lead... and I have confidence in her, but she doesn't have Lynch syndrome.

Left to myself, I may not have done it and I will be totally honest about that. It was with my wife's encouragement that we did this as a couple.
Timing of conversations about ACP in individuals with LS is critical. In our study, many participants recognized that conversations with providers and family members about ACP was important, but time was a barrier. This included a lack of time, never feeling like ACP could take precedence over other priorities, or not knowing when the timing was right. Despite the benefits, there is no clear decision about who should initiate these conversations and when they should begin.

One way to overcome this challenge is to make ACP discussions a standard of care for all patients. This will benefit every patient, but especially those with LS, who have a high chance of being diagnosed with cancer before they have an ACP discussion. One option for creating a standard of care is for all patients to have an initial discussion with their primary care provider at 18 years of age and then revisit this discussion every year during annual check-ups. A patient can choose to not have the conversation if they are not ready or are uncomfortable, or they may choose to discuss ACP with another health care provider with whom they feel more comfortable. For example, an oncologist may be better suited to have this discussion with a patient with LS or hereditary cancer. These reminders can be embedded within the electronic medical 
record and become a routine assessment, which may be less intimidating to patients. The quality of this discussion also is critical. Many participants explained that if their providers brought up ACP, they were usually just handed paperwork on the way out of their office. They did not know how to complete the paperwork or start it. A conversation is needed, which includes a discussion on why ACP is important, an overview of the different options that exist within a patient's state, and an explanation of how to properly complete the paperwork.

It is difficult for a patient to predict their own wishes in end-oflife circumstances, and literature shows that health care proxies frequently predict the wishes of their loved ones incorrectly, even after having conversations [13]. Previous literature also has shown that partners sometimes project their own wishes onto their spouses [14]. To overcome these barriers, providers should revisit ACP every year to address changes in circumstances and/or values, to ensure the patient has completed the appropriate documents, and to check that patients have shared these documents and wishes with their health care proxies. It also is important to choose the most effective documentation, and some people may find it helpful to use a document that combines both values and treatment directives, such as the Five Wishes Form [15].

Some states are taking steps toward improving the use of advance directives. One example is Michigan. The state created the Peace of Mind Registry, a free and voluntary statewide online registry, where individuals can create and store advance directive information. This registry allows secure access to individuals, health care providers, and authorized representatives [16]. Massachusetts has created Honoring Choices, an online tool which provides free ACP information. The website outlines which documents are available in Massachusetts, translates documents, and contains multilingual videos and guides to completing these documents [17]. These resources are highly valuable and demonstrate how ACP can be integrated into standard medical care.

This study presents limitations that should be considered when interpreting the results. One of these limitations is that the study used a small sample size and we recruited through social media. This typically means that the individuals in our study are more likely to be engaged in their own health care. However, one benefit of this means of recruitment is a more geographically diverse sample, with participants from every time zone in the USA [7]. As is typically the case with studies in hereditary cancer, our sample was White, female, and of higher socioeconomic status. Another limitation of this study is that because it focused on individuals with LS, the data may not necessarily be extrapolated to other populations of cancer patients. Our results evaluated one period of time in each participant's life, so it is important to consider other life circumstances that could affect these results. Lastly, this study did not survey or evaluate provider knowledge, preferences, and barriers to ACP, including the assessment of provider comfort in having these conversations with patients.
Our study findings indicate that individuals with LS complete ACP documents at about the same rate as the general population. The factors associated with completion of these documents are similar to what is seen in the broader literature. We ultimately believe ACP should be included as the standard of care for all individuals, not just those with LS, to help reduce the barriers for completion. The study findings can help to improve knowledge of ACP through educational efforts and interventions to increase the use of ACP by both providers and individuals with LS. Further studies should target a larger number of patients with LS, including a more diverse patient population. A larger, longitudinal study following patients through completion of ACP would be highly beneficial to assess how patient preferences change over time.

Funding This research was partially supported by a grant from the Collaborative Fund of the CGA (PI: A.M. Burton-Chase) as well as an ACPHS Student Summer Research Award for Victoria Fenton.

\section{Compliance with Ethical Standards}

Conflict of Interest The authors declare that they have no conflicts of interest.

\section{References}

1. Biller LH, Syngal S, Yurgelun MB. Recent advances in Lynch syndrome. Familial Cancer. 2019;18(2):211-9.

2. Giardiello FM, Allen JI, Axilbund JE, Boland CR, Burke CA, Burt $\mathrm{RW}$, et al. Guidelines on genetic evaluation and management of lynch syndrome: a consensus statement by the US Multi-Society Task Force on Colorectal Cancer. Gastroenterology. 2014;147(2):502-26.

3. Lynch HT, Lynch PM, Lanspa SJ, Snyder CL, Lynch JF, Boland CR. Review of the Lynch syndrome: history, molecular genetics, screening, differential diagnosis, and medicolegal ramifications. Clin Genet. 2009;76(1):1-18.

4. Wright AA, Zhang B, Ray A, Mack JW, Trice E, Balboni T, et al. Associations between end-of-life discussions, patient mental health, medical care near death, and caregiver bereavement adjustment. JAMA. 2008;300(14):1665-73.

5. Yadav KN, Gabler NB, Cooney E, Kent S, Kim J, Herbst N, et al. Approximately one in three US adults completes any type of advance directive for end-of-life care. Health Aff. 2017;36(7):1244-51.

6. Douma KFL, Bleeker FE, Medendorp NM, Croes EAJ, Smets EMA. Information exchange between patients with Lynch syndrome and their genetic and non-genetic health professionals: whose responsibility? J Community Genet. 2019;10(2):237-47.

7. Burton-Chase AM, Parker WM, Hennig K, Sisson F, Bruzzone L. The use of social media to recruit participants with rare conditions: Lynch syndrome as an example. JMIR Res Protoc. 2017;6(1):e12.

8. Research Electronic Data Capture. 2017; http://www.projectredcap.org. Accessed 2017-01-17.

9. Glaser BG, Strauss AL. The discovery of grounded theory: strategies for qualitative research. Chicago: Aldine Publishing; 1967.

10. Ehrenfreund M. Jahi McMath could be removed from life support despite family's wishes. 2013; https://www.washingtonpost.com/ national/jahi-mcmath-could-be-removed-from-life-supportdespite-familys-wishes/2013/12/30/41f122f4-7191-11e3-8defa33011492df2_story.html. Accessed 31 March, 2020. 
11. Petrovic P, Berve P-O, Turowski GE, Stray-Pedersen A, KramerJohansen J, Wik L. Injuries associated with mechanical chest compressions in patients with out-of-hospital cardiac arrest: a comparison of LUCAS 2 and LUCAS 2 active decompression. Circulation. 2019;140.

12. Detering KM, Hancock AD, Reade MC, Silvester W. The impact of advance care planning on end of life care in elderly patients: randomised controlled trial. Br Med J. 2010;340(7751):847.

13. Shalowitz DI, Garrett-Mayer E, Wendler D. The accuracy of surrogate decision makers: a systematic review. Arch Intern Med. 2006;166(5):493-7.

14. Moorman SM, Hauser RM, Carr D. Do older adults know their spouses' end-of-life treatment preferences? Res Aging. 2009;31(4):463-91.
15. Aging with Dignity. Five Wishes. 2020; https://fivewishes.org/. Accessed 31 March, 2020.

16. Donation GoLMOaT. Peace of Mind Registry provides opportunity for residents to document their end-of-life wishes. 2015; Peace of Mind Registry provides opportunity for residents to document their end-of-life wishes. Accessed 31 March, 2020.

17. Massachusetts HC. Your Healthcare, Your Choice; 2020; https:// www.honoringchoicesmass.com/. Accessed 31 March, 2020.

Publisher's Note Springer Nature remains neutral with regard to jurisdictional claims in published maps and institutional affiliations. 